\title{
Detrimental effects of lipopolysaccharide on the attachment and outgrowth of various trophoblastic spheroids on human endometrial epithelial cells
}

\author{
Wontae Kim ${ }^{1}$, Jungwon Choi ${ }^{2}$, Hyejin Yoon ${ }^{2}$, Jaewang Lee ${ }^{1}$, Jin Hyun Jun ${ }^{1,2}$ \\ 'Department of Biomedical Laboratory Science and ${ }^{2}$ Department of Senior Healthcare, BK21 Plus Program, Graduate School, Eulji University, \\ Seongnam, Korea
}

Objective: Lipopolysaccharide (LPS) from Gram-negative bacteria causes poor uterine receptivity by inducing excessive inflammation at the maternal-fetal interface. This study aimed to investigate the detrimental effects of LPS on the attachment and outgrowth of various types of trophoblastic spheroids on endometrial epithelial cells (ECC-1 cells) in an in vitro model of implantation.

Methods: Three types of spheroids with JAr, JEG-3, and JAr mixed JEG-3 (JmJ) cells were used to evaluate the effect of LPS on early implantation events. ECC-1 cells were treated with LPS to mimic endometrial infection, and the expression of inflammatory cytokines and adhesion molecules was analyzed by quantitative real-time polymerase chain reaction and western blotting. The attachment rates and outgrowth areas were evaluated in the various trophoblastic spheroids and ECC-1 cells treated with LPS.

Results: LPS treatment significantly increased the mRNA expression of inflammatory cytokines (CXCL1, IL-8, and IL-33) and decreased the protein expression of adhesion molecules (ITG $\beta 3$ and ITG $\beta 5$ ) in ECC-1 cells. The attachment rates of JAr and JmJ spheroids on ECC-1 cells significantly decreased after treating the ECC-1 cells with 1 and $10 \mu \mathrm{g} / \mathrm{mL}$ LPS. In the outgrowth assay, JAr spheroids did not show any outgrowth areas. However, the outgrowth areas of JEG-3 spheroids were similar regardless of LPS treatment. LPS treatment of JmJ spheroids significantly decreased the outgrowth area after 72 hours of coincubation.

Conclusion: An in vitro implantation model using novel JmJ spheroids was established, and the inhibitory effects of LPS on ECC-1 endometrial epithelial cells were confirmed in the early implantation process.

Keywords: Attachment; Implantation; In vitro; Lipopolysaccharide; Outgrowth; Spheroids

\section{Introduction}

Successful implantation depends on communication between the

Received: February 17, 2021 · Revised: April 13, 2021 · Accepted: April 21, 2021

Corresponding author: Jin Hyun Jun

Department of Biomedical Laboratory Science, Eulji University, 553 Sanseongdaero, Sujeong-gu, Seongnam 13135, Korea

Tel: +82-31-740-7210 Fax: +82-31-740-7354 E-mail:junjh55@hanmail.net

*This study was supported by the Basic Science Research Program through the National Research Foundation of Korea (NRF), funded by the Ministry of Education, Science, and Technology (2018R1D1A1B07046419 and 2020R1F1A1071918).

This is an Open Access article distributed under the terms of the Creative Commons Attribution Non-Commercial License (http://creativecommons.org/licenses/by-nc/4.0/) which permits unrestricted non-commercial use, distribution, and reproduction in any medium, provided the original work is properly cited. trophoblast of the blastocyst and the epithelial cells of the uterine endometrium. This communication is regulated by several inflammatory cytokines and adhesion molecules. Collectively, the process of implantation poses an immune challenge between the embryo (as a semi-allogenic) body and the uterus [1-3]. The maternal immune system promotes immune tolerance toward the embryo to maintain a normal pregnancy while defending against infection. The maternal immune response involves maintaining uterine receptivity, mediated by endometrial epithelial attachment molecules, trophoblast invasion, and extracellular matrix (ECM) breakdown $[4,5]$.

Hormones, growth factors, and cytokines are crucial for the maintenance of uterine receptivity and implantation stages. Various cytokines, such as interleukin, leukemia inhibitory factor, and transforming growth factor-beta, are sensitive to local and systemic changes. 
They need to be appropriately regulated for successful implantation $[6,7]$. These cytokines are mostly mediated by the toll-like receptor (TLR) family, which is the main regulator of the immune response. Human endometrial tissue and trophoblasts express the TLR family [8]. The 10 membrane-spanning members of the TLR family play a critical role in modulating the inflammatory responses in humans. Each receptor responds to a specific ligand. Lipopolysaccharide (LPS) is a well-known endotoxin that consists of a lipid and a polysaccharide composed of O-antigen, outer core, and inner core joined by a covalent bond. LPS is a component of the cell wall of Gram-negative bacteria (e.g., Escherichia coli, Ureaplasma urealyticum, and Gardnerella vaginalis) $[9,10]$. LPS is an antigen that induces immune responses in uterine endometrial cells by TLR4 activation. LPS can trigger an imbalance in cytokines in the uterine endometrium [11,12].

Endometrial epithelial cells secrete hormones (estrogen and progesterone), growth factors, and various types of cytokines to enable successful implantation [13]. Adhesion molecules secreted by endometrial epithelial cells are an important factor in the embryo attachment stage $[6,14]$. Interactions between cells and the ECM are mediated by adhesion molecules [15], which consist of four subtypes: immunoglobulins, cadherins, integrins (ITGs), and selectins. ITGs are a major class of receptors within the ECM that mediate cell-ECM interactions with collagen, fibrinogen, fibronectin, and vitronectin. ITGs provide essential links between the extracellular environment and intracellular signaling pathways, and are transmembrane receptors that mediate cell adhesion $[16,17]$. ITGs consist of two subunits: alpha ( $\alpha$ ) and beta ( $\beta$ ). In humans, ITGs have 18 a subunits and $8 \beta$ subunits [18]. In this study, we analyzed ITGaV, ITG $\beta 3$, and ITG $\beta 5$ to understand the effect of LPS on the attachment of trophoblastic spheroids to uterine endometrial epithelial cells.

Many in vitro models have provided insights into the implantation process $[19,20]$. In a recent study, in vitro implantation models were designed using the spheroid form of trophoblastic cells to surrogate embryos [21]. Trophoblast cell lines, including JAr, JEG-3, and human endometrial epithelial cells of ECC-1 were used as in vitro implantation models in previous studies [22-24]. This study was performed to investigate the detrimental effects of LPS on the attachment and outgrowth of various types of trophoblastic spheroids and endometrial epithelial cells as an in vitro model of implantation.

\section{Methods}

\section{Culture of human trophoblastic and endometrial epithelial cells}

The human trophoblastic JEG-3 cell line was cultured in DMEM (Welgene, Gyeongsan, Korea) and supplemented with 10\% fetal bovine serum (FBS; Gibco, Waltham, MA, USA) and 1\% penicillin-strep- tomycin (P/S; Lonza, Morristown, NJ, USA). The human trophoblastic JAr cell line and human endometrial epithelial cells (ECC-1 cells) were cultured in RPMI 1640 (Welgene) with 10\% FBS and 1\% P/S. Cells were cultured under standard conditions $\left(37^{\circ} \mathrm{C}, 5 \% \mathrm{CO}_{2}\right)$, and the culture medium was replaced with fresh medium every 48 hours. LPS from E. coli 0111: B4 (Sigma-Aldrich, St. Louis, MO, USA) was used to treat the ECC-1 cells to mimic endometrial infections caused by Gram-negative bacteria.

\section{Preparation of various trophoblastic spheroids using the hanging drop method and the Organoid 3D culture kit}

Spheroids of JAr, JEG-3, and JAr mixed JEG-3 (JmJ) were prepared using the hanging drop method and the Organoid 3D culture kit (Cell Smith, Seoul, Korea). JAr and JEG-3 cells were mixed at a 1:1 ratio to prepare JmJ spheroids. Using the hanging drop method, trophoblast cells $\left(2 \times 10^{4} / 20 \mu \mathrm{L}\right.$ drop) were plated onto the lid of a 100 -mm dish in a regular array (20 drops/lid). The lid was inverted over the bottom, which was filled with Dulbecco's phosphate-buffered saline (DPBS; Biowest, Riverside, MO, USA), and cultured under standard conditions for 72 hours. Using the Organoid 3D culture kit, cells were seeded at a density of $1 \times 10^{5}$ cells in $3 \mathrm{~mL}$ of the medium. The cells were incubated for 72 hours in the Organoid 3D culture kit. Spheroids sized between 200 and $300 \mu \mathrm{m}$ were selected and used for subsequent experiments. The morphology of spheroids cultured by the different methods was observed using an EVOS XL Core Cell Imaging System (Thermo-Fisher, Waltham, MA, USA).

\section{Histology of various types of trophoblastic spheroids}

Three types of trophoblastic spheroids were fixed with $4 \%$ paraformaldehyde (Biosesang, Seongnam, Korea), and over 50 spheroids were suspended in $20 \mu \mathrm{L}$ of $2 \%$ agarose gel solution. The pre-embedded agarose gel blocks were embedded in paraffin, and sections of paraffin blocks with a thickness of $6 \mu \mathrm{m}$ were cut. The sections were placed on glass slides and stained with hematoxylin and eosin (H\&E). The spheroids were observed under a Nikon Eclipse 80i microscope.

\section{Quantitative analysis of mRNA expression in ECC-1 cells treated with LPS}

Total RNA from the ECC-1 endometrial epithelial cells was isolated using TRIzol reagent (Ambion, Austin, TX, USA). Complementary DNA was synthesized using a cDNA reverse transcription kit (Takara Bio, Shiga, Japan). All quantitative SYBR-based quantitative real-time polymerase chain reaction (qRT-PCR) assays were performed in a 20 $\mu \mathrm{L}$ reaction volume using the StepOne software ver. 2.3 using the SYBR Green master mix, SensiFAST SYBR Hi-ROX Kit (Bioline, London, England), and $10 \mathrm{pM}$ of each specific primer and $1 \mu \mathrm{L}$ of CDNA per re- 
action (Table 1). Each qRT-PCR involved an initial denaturation step at $95^{\circ} \mathrm{C}$ for 10 minutes, followed by 40 cycles of denaturation at $95^{\circ} \mathrm{C}$ for 15 seconds, annealing at a specific temperature of each primer for 30 seconds, and extension at $72^{\circ} \mathrm{C}$ for 30 seconds. Finally, quantitative analysis was performed using the $2^{-\Delta \Delta c t}$ method with $\beta$-actin as an internal control [25].

\section{Western blot analysis of ECC-1 cells treated with LPS}

Equal amounts of total protein $(20 \mu \mathrm{g})$ from ECC- 1 cells treated with LPS were separated by sodium dodecyl sulfate-polyacrylamide gel electrophoresis and transferred to nitrocellulose membranes. The membranes were blocked with 5\% skim-milk/TBS-T (Tris-buffered saline-Tween 20) solution (Bio-Rad, Contra Costa, CA, USA) and incubated with anti-ITGaV (ab179475), ITG $\beta 3$ (ab197662), ITG $\beta 5$ (ab31327; Abcam, Cambridge, England), and anti-GAPDH (SC-32233; Santa Cruz Biotechnology, Dallas, TX, USA) antibodies. After the reaction with appropriate secondary antibodies linked to horseradish peroxide (Abcam), the signals were visualized using the ChemiDoc MP Imaging System (Bio-Rad). Densitometry was performed using ImageJ software (National Institutes of Health, Bethesda, MD, USA). Relative optical density was calculated by dividing the optical density of ITG protein by that of the internal control (GAPDH).

\section{Evaluation of the attachment rate and outgrowth of trophoblastic spheroids on ECC-1}

Endometrial epithelial cells of ECC-1 were cultured until confluence in 775 flasks and then detached using $0.25 \%$ trypsin/EDTA. Approximately $1 \times 10^{5}$ endometrial cells were seeded in 12-well plate culture dishes and cultured at $37^{\circ} \mathrm{C}$ until $100 \%$ confluency. After LPS treatment in ECC-1 $(0,1$, and $10 \mu \mathrm{g} / \mathrm{mL}$ of LPS, incubated for 24 hours), trophoblastic spheroids were added to ECC-1 and co-cultured for 6 hours. The attachment rate of trophoblastic spheroids to endometrial epithelial cells was evaluated at 0, 1, 2, 4, and 6 hours. The number of attached spheroids was counted by tapping by hand 3-5 times. After 72 hours of co-culture, the outgrowth area and spheroid area were analyzed using ImageJ software (National Institutes of Health). The areas of outgrowth of the spheroids were measured in pixel units. The ratio of the outgrowth area (outgrowth area/spheroid area) was calculated and analyzed.

\section{Statistical analysis}

All experiments were performed at least in triplicate, and more than 250 spheroids were used in each group. Data are presented as mean \pm standard error of the mean. The statistical significance of differences between groups was analyzed using one-way analysis of variance and the Tukey test. Data were analyzed using Prism GraphPad software ver. 5.0 (GraphPad, San Diego, CA, USA). Statistical significance was set at $p<0.05$.

\section{Results}

\section{Morphology and histology of trophoblastic spheroids using the hanging drop method and Organoid 3D culture kit}

Microscopic analysis of the various types of spheroids was performed to obtain more detailed observations of their morphology. Spheroid formation and cell distribution were observed histologically by H\&E staining. The spheroids cultured by both methods (hanging drop and the Organoid 3D culture kit) were well prepared for the in vitro implantation model. All trophoblastic spheroids were maintained at a high cell density (Figures 1 and 2).

Table 1. Primer sequences of inflammatory cytokines, adhesion molecules, and internal controls

\begin{tabular}{|c|c|c|c|c|}
\hline Gene & Primer sequence & Product size & GenBank accession number & Annealing temperature $\left({ }^{\circ} \mathrm{C}\right)$ \\
\hline \multirow[t]{2}{*}{ CXCL1 } & F:CACCTGGATTGTGCCTAATGT & $273 \mathrm{bp}$ & NM_001511.4 & 60 \\
\hline & R:TTGCAGGCTCCTCAGAAATA & & & \\
\hline \multirow[t]{2}{*}{ IL-8 } & $\mathrm{F}: \mathrm{GGCACAAACTTTCAGAGACAG}$ & $153 \mathrm{bp}$ & NG_029889.1 & 60 \\
\hline & $\mathrm{R}: \mathrm{ACACAGAGCTGCAGAAATCAGG}$ & & & \\
\hline \multirow[t]{2}{*}{ IL-33 } & F:GTGACGGTGTTGATGGTAAGA & $349 \mathrm{bp}$ & NM_001314044.2 & 60 \\
\hline & R:CCTTCTCCAGTGGTAGCATTT & & & \\
\hline \multirow[t]{2}{*}{$I T G \alpha V$} & $\mathrm{~F}:$ AATCTTCCAATTGAGGATATCAC & $140 \mathrm{bp}$ & NM_001145000.3 & 61 \\
\hline & R:AAAACAGCCAGTAGCAACAAT & & & \\
\hline \multirow[t]{2}{*}{ ITG $\beta 3$} & F:AGTCAGGGAGAGCTGAACTA & $294 \mathrm{bp}$ & NM_000212.3 & 60 \\
\hline & R:GGGTGTGGAATTAGGAGGTAAA & & & \\
\hline \multirow[t]{2}{*}{ ITG $\beta 5$} & F:TAGGTAGGCACCACAGAGAA & $219 \mathrm{bp}$ & NM_002213.5 & 60 \\
\hline & R:CAGCCCAGCATCTCAGTATTT & & & \\
\hline \multirow[t]{2}{*}{$\beta$-actin } & $\mathrm{F}:$ CATGTACGTTGCTATCCAGGC & $250 \mathrm{bp}$ & NM_001101.5 & 60 \\
\hline & R: CTCCTTAATGTCACGCACGAT & & & \\
\hline
\end{tabular}

CXCL1, CXC motif ligand 1; IL, interleukin; ITG, integrin; $F$, forward; $R$, reverse. 

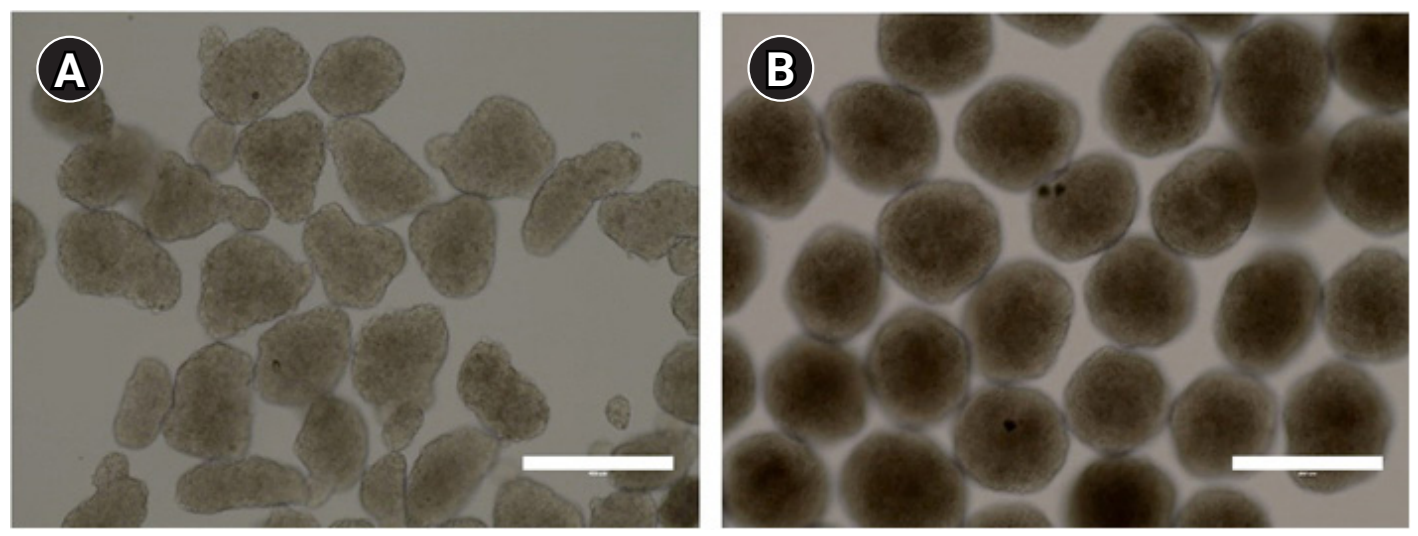

Figure 1. Microscopic morphology of JAr mixed JEG-3 trophoblastic spheroids generated by the hanging drop and organoid kit method. Spheroids were observed by EVOS XL Core Cell Imaging System (Thermo-Fisher). Representative spheroids generated (A) by the hanging drop method and (B) by the Organoid 3D cell culture kit method. Scale bar $=400 \mu \mathrm{m}$.
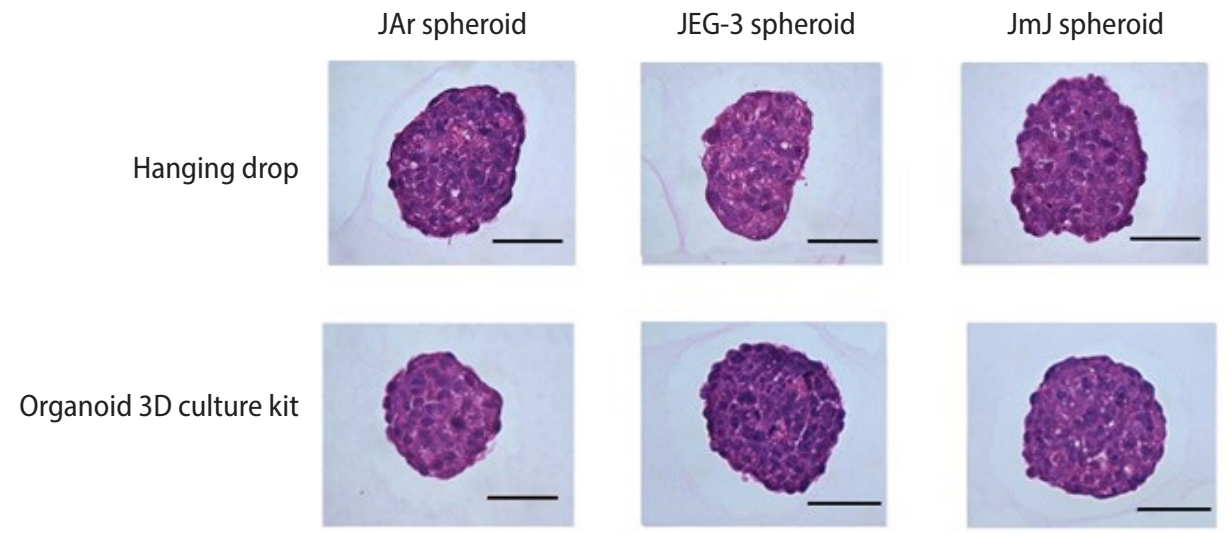

$J \mathrm{~m} J$ spheroid

Figure 2. Histological characteristics of the various types of trophoblastic spheroids (JAr, JEG-3, and JAr mixed JEG-3 [JmJ]) in different culture methods. Histological characteristics of various spheroids by H\&E staining observed with a Nikon Eclipse 80i microscope. The upper images of trophoblastic spheroids were formed by the hanging drop method, and the lower image by the Organoid 3D culture kit method. Scale bar $=200 \mu \mathrm{m}$.

\section{Effect of LPS on mRNA expression of adhesion molecules and inflammatory cytokines in ECC-1 cells}

To evaluate the effect of LPS on ECC-1 cells, the expression of inflammatory cytokines and adhesion molecules was analyzed by qRTPCR. LPS treatment significantly increased the mRNA expression of $C X C L 1$, IL-8, and IL-33 and decreased ITG $\beta 3$ and ITG $\beta 5$ expression in ECC-1 cells $(p<0.05)$. However, the mRNA expression of ITGaV in endometrial epithelial cells was not changed by LPS treatment (Figure 3).

\section{Effect of LPS on protein expression of adhesion molecules in ECC-1}

To evaluate the effect of LPS on ECC-1, the expression of adhesion molecules (ITGaV, ITG $\beta 3$, and ITG $\beta 5$ ) was analyzed by western blotting. ITG $\beta 3$ and ITG $\beta 5$ expression decreased in response to LPS treatment. However, ITGaV expression was not significantly different between the control and LPS-treated groups (Figure 4).

\section{Effect of LPS on the attachment of various trophoblastic spheroids on ECC- 1}

The attachment rate between trophoblastic spheroids and the ECC-1 endometrial epithelial cells was evaluated in a time-dependent manner. The attachment rate was evaluated under $1 \%$ FBS or without FBS. LPS treatment did not affect the attachment rate of JEG-3 spheroids (Table 2). However, the attachment rates of JAr spheroids to ECC-1 cells were significantly decreased by LPS treatment $(p<0.05)$. The attachment rates of JAr spheroids on ECC -1 were similar in the LPS-treated and control groups after 6 hours of co-culture (Table 3). In the absence of FBS supplementation, the attachment rate was significantly decreased by LPS treatment compared to the condition with $1 \%$ FBS supplementation $(p<0.05)$. In the following experiments, the attachment rate of JmJ spheroids was analyzed in the absence of FBS supplementation. In JmJ spheroids, the attachment rate of LPS treatment was significantly lower than that of the 
control after 2 hours of co-culture, as shown in Table $4(p<0.05)$.

\section{Effect of LPS on the outgrowth of various trophoblastic spheroids on ECC- 1 cells}

The outgrowth of trophoblastic spheroids on the ECC-1 endome-

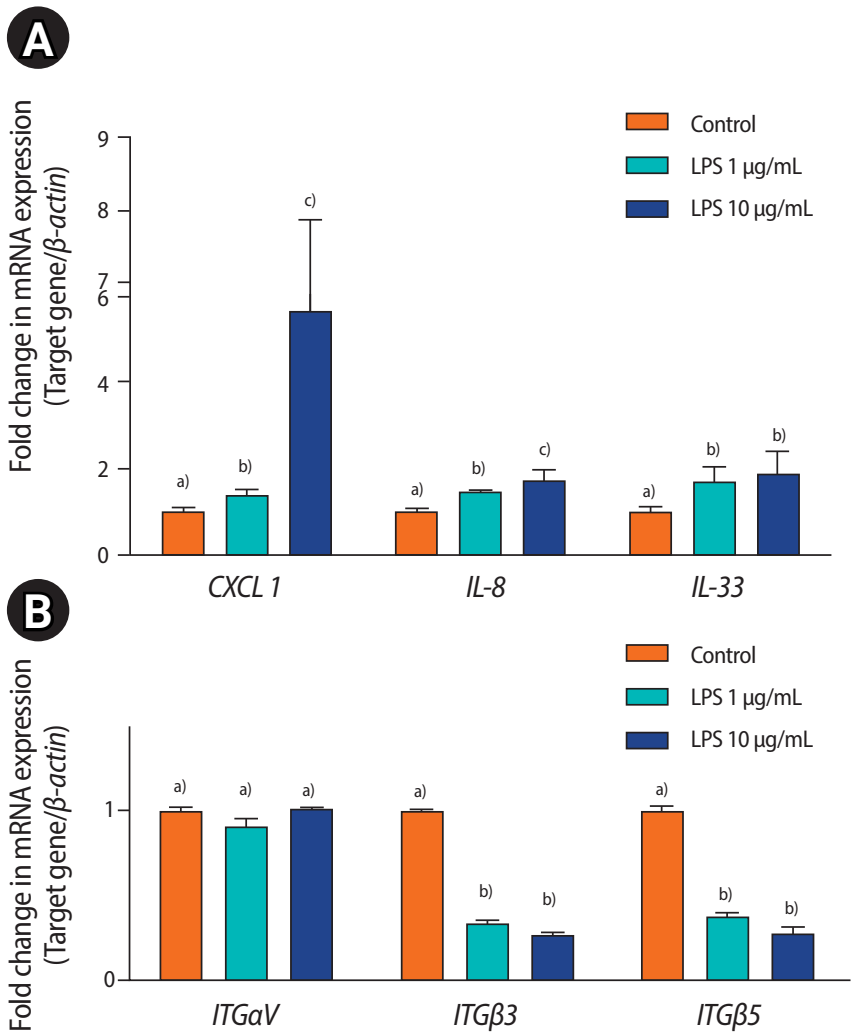

Figure 3. Quantitative analysis of mRNA expression in ECC-1 cells treated with lipopolysaccharide (LPS). Quantitative real-time polymerase chain reaction analysis of inflammatory cytokines $(A)$ and adhesion molecules (B) in ECC-1 cells treated by LPS. Values are presented as mean \pm standard error of the mean. CXCL1, CXC motif

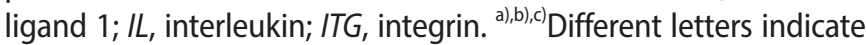
significant differences (one-way analysis of variance and the Tukey test, $p<0.05)$. trial epithelial cells was evaluated after 72 hours of co-culture. The JAr spheroids did not show any outgrowth area, whereas the JEG-3 and JmJ spheroids showed outgrowth areas on ECC-1. Nevertheless, LPS treatment did not affect the outgrowth area of JEG-3 spheroids. Interestingly, LPS treatment significantly decreased the outgrowth area of JmJ on ECC- 1 cells after 72 hours of co-culture, as shown in Figure 5 (control, $1.90 \pm 0.06 ; 1 \mu \mathrm{g} / \mathrm{mL}$ LPS, $1.62 \pm 0.06$; and $10 \mu \mathrm{g} / \mathrm{mL}$ LPS, $1.64 \pm 0.06 ; p<0.05)$.

\section{Discussion}

Implantation is a complex immunological process. The inflammatory environment of the uterus changes from a pro-inflammatory to an anti-inflammatory state throughout implantation and pregnancy. A strong inflammatory response is necessary during implantation [26-30]. However, an imbalance in the immune status of the uterus can cause serious problems such as implantation failure and pregnancy loss [31,32]. In this study, activation of TLR4 in ECC-1) by LPS significantly reduced the attachment rate and outgrowth area between trophoblastic spheroids and endometrial epithelial cells.

It has been shown that implantation failure is induced by LPS through stimulation of the innate immune system and activation of the TLR4 pathway in the early stages of pregnancy [11,33]. A similar study suggested that TLR3 stimulated by a synthetic ligand, poly I: C (which is a double-stranded RNA molecule) in CBA/J female mice increased fetal losses [34] and reduced actin polymerization and adhesion molecule expression in endometrial cells [35]. In another study, activation of TLR5 in a human telomerase immortalized endometrial epithelial cell line (hTERT-EECs) by bacterial flagellin significantly decreased the attachment rate between JAr spheroids and underlying endometrial cells [36]. This study suggested that activation of the TLR family by various antigens at early stages of pregnancy could induce detrimental effects on implantation.

An endometrial epithelial cell line, ECC-1, was treated with LPS to
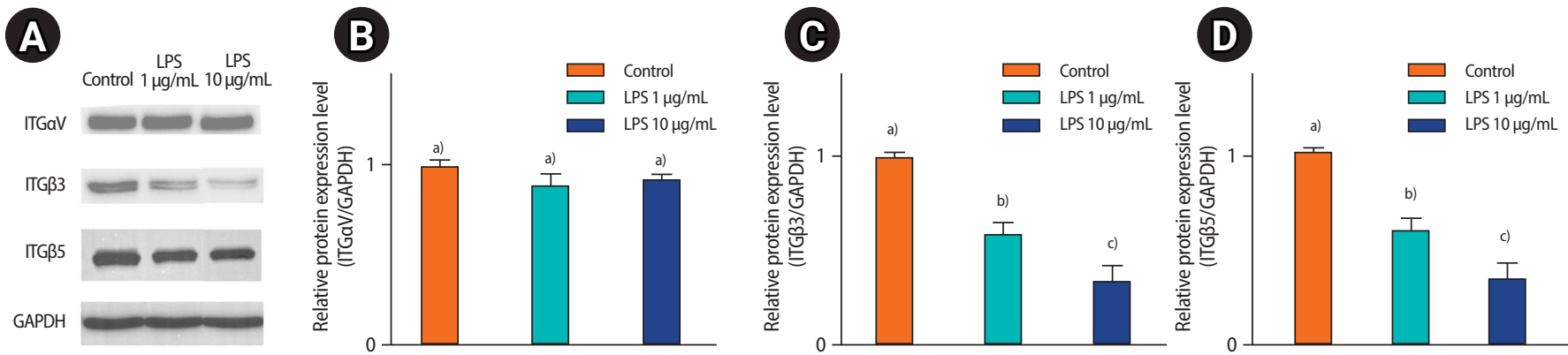

Figure 4. (A) Western blot analysis of ITGaV, ITG 33 , and ITG 35 in ECC-1 cells treated with lipopolysaccharide (LPS). Western blot analysis of ITGaV, ITGß3, ITG $\beta 5$, and GAPDH. Densitometry data of (B) ITGaV, (C) ITG 3 , and (D) ITG $\beta 5$. Values are presented as mean \pm standard error of the mean. ${ }^{\mathrm{a}, b), \mathrm{c}}$ Different letters indicate significant differences (one-way analysis of variance and the Tukey test, $p<0.05$ ). 
Table 2. Attachment rate of JEG-3 spheroids on ECC-1 treated by LPS

\begin{tabular}{lccc}
\hline Variable & Control (\%) & LPS $(1 \mu \mathrm{g} / \mathrm{mL})(\%)$ & $\mathrm{LPS}(10 \mu \mathrm{g} / \mathrm{mL})(\%)$ \\
\hline With FBS (1\%) & & & \\
$1 \mathrm{hr}$ & $50.9 \pm 3.4$ & $52.1 \pm 1.8$ & $49.8 \pm 6.0$ \\
$2 \mathrm{hr}$ & $69.3 \pm 0.7$ & $73.8 \pm 5.1$ & $73.9 \pm 7.3$ \\
$4 \mathrm{hr}$ & $97.1 \pm 2.9$ & $92.5 \pm 6.1$ & $96.3 \pm 3.1$ \\
$6 \mathrm{hr}$ & 100.0 & 100.0 & 100.0 \\
Without FBS & & & \\
$1 \mathrm{hr}$ & $54.8 \pm 2.9$ & $45.6 \pm 4.4$ & $62.9 \pm 7.6$ \\
$2 \mathrm{hr}$ & $69.2 \pm 0.4$ & $66.4 \pm 7.5$ & $72.9 \pm 8.4$ \\
$4 \mathrm{hr}$ & $93.1 \pm 3.8$ & $96.6 \pm 2.2$ & $99.1 \pm 1.0$ \\
$6 \mathrm{hr}$ & $96.7 \pm 4.1$ & $98.3 \pm 1.7$ & 100.0 \\
\hline
\end{tabular}

Values are presented as mean \pm standard error of the mean (\%). The attachment rate was evaluated in the condition with $1 \%$ FBS or without FBS supplementation.

LPS, lipopolysaccharide; FBS, fetal bovine serum.

Table 3. Attachment rate of JAr spheroids on ECC-1 treated by LPS

\begin{tabular}{lccc}
\hline Variable & Control (\%) & LPS $(1 \mu \mathrm{g} / \mathrm{mL})(\%)$ & LPS $(10 \mu \mathrm{g} / \mathrm{mL})(\%)$ \\
\hline With FBS (1\%) & & & \\
$1 \mathrm{hr}$ & $55.7 \pm 2.7^{\mathrm{a})}$ & $37.4 \pm 2.9^{\mathrm{b})}$ & $36.0 \pm 2.1^{\mathrm{b})}$ \\
$2 \mathrm{hr}$ & $81.7 \pm 4.5^{\mathrm{a})}$ & $61.7 \pm 5.9^{\mathrm{b})}$ & $56.0 \pm 7.3^{\mathrm{b})}$ \\
$4 \mathrm{hr}$ & $90.3 \pm 2.9^{\mathrm{a})}$ & $90.9 \pm 1.9^{\mathrm{a})}$ & $81.3 \pm 2.1^{\mathrm{b})}$ \\
$6 \mathrm{hr}$ & $97.7 \pm 1.4^{\mathrm{a})}$ & $98.1 \pm 1.3^{\mathrm{a}}$ & $84.1 \pm 6.1^{\mathrm{b})}$ \\
Without FBS & & & \\
$1 \mathrm{hr}$ & $43.2 \pm 3.0^{\mathrm{a})}$ & $33.0 \pm 3.5^{\mathrm{b})}$ & $28.2 \pm 4.0^{\mathrm{b}}$ \\
$2 \mathrm{hr}$ & $69.2 \pm 3.5^{\mathrm{a})}$ & $55.4 \pm 3.4^{\mathrm{b}}$ & $50.1 \pm 4.2^{\mathrm{c}}$ \\
$4 \mathrm{hr}$ & $90.1 \pm 2.0^{\mathrm{a})}$ & $78.4 \pm 1.1^{\mathrm{b}}$ & $75.0 \pm 3.2^{\mathrm{b})}$ \\
$6 \mathrm{hr}$ & $98.3 \pm 1.4^{\mathrm{a}}$ & $93.9 \pm 2.1^{\mathrm{a})}$ & $89.2 \pm 4.9^{\mathrm{a})}$ \\
\hline
\end{tabular}

Values are presented as mean \pm standard error of the mean (\%). The attachment rate was evaluated in the condition with $1 \%$ FBS or without FBS supplementation.

LPS, lipopolysaccharide; FBS, fetal bovine serum.

a),b,c) Different superscript letters indicate statistically significant differences $(p<0.05)$ by one-way analysis of variance and Tukey test.

Table 4. Attachment rate of JmJ spheroids on ECC-1 treated by LPS

\begin{tabular}{lccc}
\hline Without FBS & Control (\%) & LPS $(1 \mu \mathrm{g} / \mathrm{mL})(\%)$ & LPS $(10 \mu \mathrm{g} / \mathrm{mL})(\%)$ \\
\hline $1 \mathrm{hr}$ & $31.2 \pm 2.7^{\mathrm{a}}$ & $19.5 \pm 2.8^{\mathrm{b})}$ & $13.6 \pm 1.8^{\mathrm{b})}$ \\
$2 \mathrm{hr}$ & $\left.48.5 \pm 1.7^{\mathrm{a}}\right)$ & $38.4 \pm 1.3^{\mathrm{b})}$ & $36.4 \pm 1.1^{\mathrm{b})}$ \\
$4 \mathrm{hr}$ & $77.3 \pm 2.2^{\mathrm{a})}$ & $71.5 \pm 3.6^{\mathrm{a})}$ & $73.3 \pm 5.5^{\mathrm{a})}$ \\
$6 \mathrm{hr}$ & $97.2 \pm 0.8^{\mathrm{a})}$ & $96.6 \pm 1.1^{\text {a) }}$ & $98.4 \pm 0.9^{\mathrm{a})}$ \\
\hline
\end{tabular}

Values are presented as mean \pm standard error of the mean (\%). The attachment rate was evaluated without FBS.

JmJ, JAr mixed JEG-3; LPS, lipopolysaccharide; FBS, fetal bovine serum.

a),b) Different superscript letters indicate statistically significant differences $(p<0.05)$ by one-way analysis of variance and Tukey test.

mimic endometrial infections caused by Gram-negative bacteria. In the implantation process, LPS affects not only endometrial epithelial cells, but also competent blastocysts [37]. LPS stimulates the TLR4

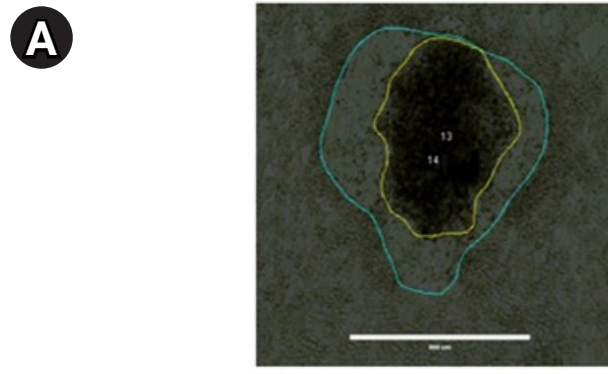

ROA: blue line/yellow line

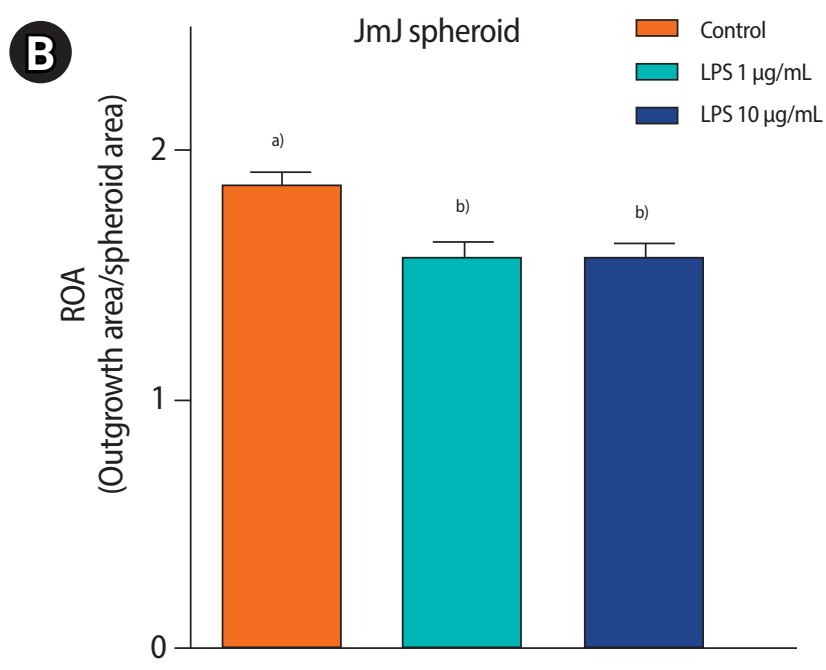

Figure 5. Ratio of the outgrowth area (ROA) of JAr mixed JEG-3 $(\mathrm{JmJ})$ spheroids on ECC-1 cells treated with lipopolysaccharide (LPS). Outgrowth areas of spheroids were measured after 72 hours of coculture. (A) The ROA (outgrowth area [blue line]/spheroid area [yellow line]) was calculated with ImageJ. (B) Mean ROA of three groups were represented. Each experiment was performed at least three times, and more than 110 spheroids were used in each group. Values are presented as mean \pm standard error of the mean. ${ }^{\mathrm{a}, \mathrm{b})}$ Different letters indicate significant differences (one-way analysis of variance and the Tukey test, $p<0.05$ ).

pathway to activate the inflammatory response [38]. TLR4 is essential for LPS activation. However, the JAr and JEG-3 human trophoblast cell lines do not express TLR4 on the membrane [39]. Other regulated pathways could potentially affect trophoblasts, resulting in the detrimental effect of LPS. Human endometrial epithelial cell lines (Ishikawa and ECC-1 cells) expressing TLR4 were used to evaluate the attachment rate of trophoblastic spheroids by LPS treatment [40]. In a pilot study, endometrial epithelial cell lines (Ishikawa and ECC-1 cells) were used in the implantation model to investigate the effects of LPS. LPS treatment did not affect the attachment rate between Ishikawa cells and trophoblastic spheroids. However, the attachment rate between ECC-1 cells and trophoblastic spheroids of JAr decreased with LPS treatment. Thus, we used EEC-1 endometrial epithelial cells in subsequent experiments of the implantation model. 
The attachment rate of JAr and JEG-3 spheroids with ECC-1 cells was evaluated in the presence of $1 \%$ FBS and without FBS supplementation. In our experiments, the detrimental effect of LPS could be more clearly observed in the absence of FBS supplementation than in the $1 \%$ FBS condition due to the fact that FBS contains various factors such as growth factors and adhesion molecules [41]. Bas et al. [42] showed that FBS contains unknown factors and can inhibit TLR activation. For this reason, we designed the attachment and outgrowth experiments of JmJ spheroids without FBS supplementation.

The spheroids prepared by both methods (hanging drop and the Organoid 3D culture kit) were observed histologically using $\mathrm{H} \& \mathrm{E}$ staining. The microscopic observations showed that the Organoid $3 \mathrm{D}$ culture kit could provide spheroids with more consistent size and circularity than the hanging drop method. Therefore, spheroids prepared with the Organoid 3D culture kit might be more reliable for growth assays that analyze the ratio of the growth area to the spheroid area. We attempted to identify distinct features of $\mathrm{JmJ}$ spheroids compared to JAr and JEG-3 spheroids by histological observations. However, no significant differences were observed between the groups.

Choriocarcinoma JAr and JEG-3 cell lines were used to establish an in vitro model to investigate the attachment of trophoblast cells. JAr spheroids were suitable for investigating the effect of LPS on the attachment rate. However, the JAr spheroids did not show any outgrowth areas, indicating that they were not suitable for outgrowth assays. The other trophoblastic spheroids, JEG-3, had a clear outgrowth area for outgrowth assays. However, LPS-treated ECC-1 cells showed no significant difference in the attachment rates of JEG-3 spheroids compared to the control group.

Notably, JmJ spheroids were suitable for both attachment rate and outgrowth assays with ECC-1 cells. The JmJ spheroids showed a significant difference in the attachment rate after treatment with LPS. Moreover, the JmJ spheroids had a clear outgrowth area, which was suitable for outgrowth assays. The JmJ spheroids were used for subsequent experiments involving LPS treatment.

In the attachment rate assay, all spheroids were attached to ECC-1 after 6 hours of co-culture. Usually, the trophoblastic cells of carcinoma cell lines are used for in vitro implantation models. Carcinoma cell lines have a stronger invasion ability than normal cell lines [43]. Taken together, using a highly invasive carcinoma cell line could mask the detrimental effects of LPS on the attachment rate after 6 hours of co-culture of trophoblastic spheroids and ECC-1.

The expression of inflammatory cytokines and adhesion molecules was analyzed in the present study. ITGs are adhesion molecules in endometrial, decidual, and extravillous cytotrophoblast cells. They participate in cell-cell adhesion and adhesion between cells and components of the ECM [44]. LPS treatment significantly decreased the mRNA expression of ITG $\beta 3$ and ITG $\beta 5$. However, the mRNA levels of ITG $\beta 1$ and ITGaV were not changed by LPS treatment. A recent study by Guo et al. [45] using a bovine model could help understand these results. The expression of ITG $\beta 3$, ITG $\beta 5$, ITG $\beta 7$, and most transcripts coding for cell adhesion molecules (PCDH7, PKP1, PKP3, CT, CTNNA3, CTNNAL1, and $C D H 2$ ) were downregulated after treatment with $2 \mu \mathrm{g} / \mathrm{mL}$ LPS in bovine endometrial epithelial cells. However, transmembrane glycoproteins that mediate cell-cell interactions through calcium binding (i.e., ITGB6, CDH26, ITGAV, and CELSR1) were overexpressed after LPS treatment [46]. In a preliminary study, we evaluated the relative expression of ITG $\beta 3$ and ITG $\beta 5$ mRNA to determine whether LPS treatment might decrease the attachment between spheroids and ECC-1 (data not shown). However, there were no significant differences among the different types of spheroids (JAr, JEG-3, and JmJ).

In this study, the mRNA expression of the inflammatory cytokines CXCL1, IL-8, and IL-33 was higher in LPS-treated ECC-1 cells. CXCL1 and IL-8 are representative inflammatory cytokines in endometrial cells exposed to LPS. IL-8 and CXCL1 are cytokines with neutrophil chemotactic and activating activity and $\mathrm{T}$ cell chemotactic activity that play a major role in the recruitment of leukocytes to the endometrium via the CXCR1 and CXCR2 pathways [46-48]. However, the relationship between IL-33 and LPS in endometrial epithelial cells remains unclear. In a study by Miller et al. [49], IL-33 stimulated the expression of inflammatory cytokines, such as CXCL1, IL-6, and IL-15, in the progression of endometriosis. Further studies are needed to understand the relationship between the inflammatory response in endometrial cells and IL-33 expression.

Our study had some limitations. Numerous factors are involved in the implantation process in vivo, including immune cells, stromal cells, and hormones [50]. However, in this study, only trophoblastic cells and endometrial epithelial cells were used because of the limitations of the in vitro model. Including various other factors in the in vitro model would help to understand the details of the implantation process.

This study had several advantages. The implantation process is highly complex and difficult to mimic in vitro. Many researchers have used embryo transfer to assess implantation potential. However, embryo transfer into pseudopregnant mice requires expert experimental skills and numerous sacrificial animals [51-53]. An in vitro implantation model using the novel trophoblastic JmJ spheroids and ECC-1 endometrial epithelial cells could overcome these problems.

The findings of our study could help to understand the detrimental effects of LPS on the attachment and outgrowth of various types of trophoblastic spheroids and endometrial epithelial cells in an in vitro model of implantation (Figure 6). The results of this study suggest that alterations in the expression levels of inflammatory cytokines 
A

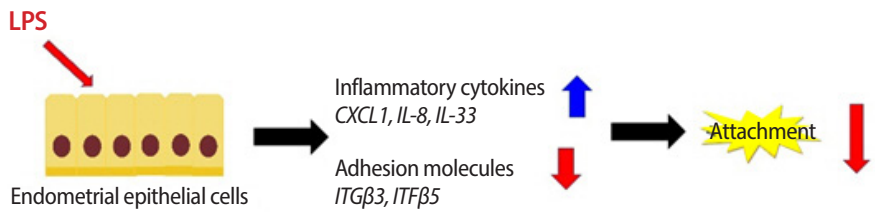

B

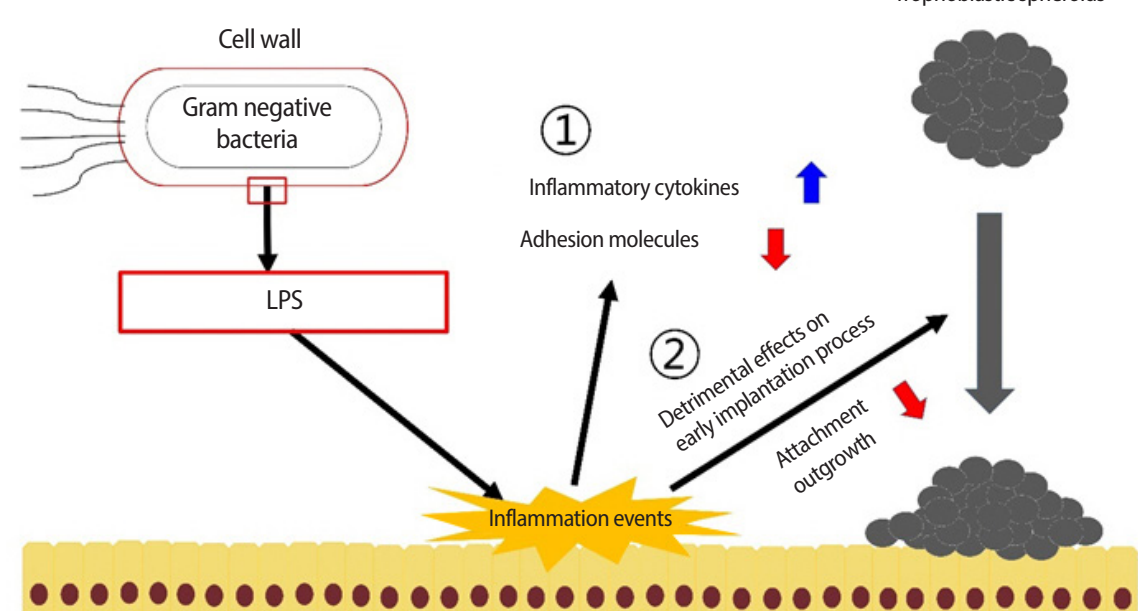

Endometrial epithelial cells

Figure 6. Schematic representation of the effects of lipopolysaccharide (LPS) on the attachment and outgrowth of trophoblastic spheroids on ECC-1 endometrial epithelial cells. (A) Gene expression changes of inflammatory cytokines (CXCL1, IL-8, and IL-33) and cell adhesion molecules (ITG $\beta 3$, ITG $\beta 5)$ were confirmed by quantitative real-time polymerase chain reaction and Western blotting after LPS treatment in ECC-1 endometrial epithelial cells. (B) Treatment with LPS changed the inflammatory cytokines and cell adhesion molecules. The detrimental effect of LPS was shown by a decrease in the attachment rate and outgrowth area of trophoblastic spheroids on endometrial epithelial cells. CXCL1, CXC motif ligand 1; IL, interleukin; ITG, integrin.

(CXCL1, IL-8, and IL-33) and adhesion molecules (ITG $\beta 3$ and ITG $\beta 5$ ) by LPS treatment might be related to reduced trophoblastic spheroid attachment and outgrowth on endometrial epithelial cells.

\section{Conflict of interest}

Jin Hyun Jun is an Associate Editor of the journal, but he was not involved in the peer reviewer selection, evaluation, or decision process of this article. No other potential conflicts of interest relevant to this article were reported.

\section{ORCID}

Wontae Kim Jungwon Choi

Hyejin Yoon

Jaewang Lee Jin Hyun Jun https://orcid.org/0000-0001-5711-3952 https://orcid.org/0000-0003-0027-2068 https://orcid.org/0000-0003-1467-5749 https://orcid.org/0000-0001-6801-7149 https://orcid.org/0000-0001-9898-4471

\section{Author contributions}

Conceptualization: JL, JHJ. Data curation: WK, JC, HY. Formal analysis: WK. Funding acquisition: JL, JHJ. Methodology: WK, JC. Project administration: WK, HY. Visualization: WK, JC. Writing-original draft: WK. Writing-review \& editing: JL, JHJ.

\section{References}

1. Salamonsen LA, Hannan NJ, Dimitriadis E. Cytokines and chemokines during human embryo implantation. roles in implantation and early placentation. Semin Reprod Med 2007;25:437-44.

2. Egashira M, Hirota Y. Uterine receptivity and embryo-uterine interactions in embryo implantation: lessons from mice. Reprod Med Biol 2013;12:127-32.

3. Kim GJ. Role of trophoblast in implantation and placenta development. Clin Exp Reprod Med 2010;37:181-9.

4. Staun-Ram E, Shalev E. Human trophoblast function during the implantation process. Reprod Biol Endocrinol 2005;3:56.

5. Ander SE, Diamond MS, Coyne CB. Immune responses at the ma- 
ternal-fetal interface. Sci Immunol 2019;4:eaat6114.

6. Guzeloglu-Kayisli O, Kayisli UA, Taylor HS. The role of growth factors and cytokines during implantation. endocrine and paracrine interactions. Semin Reprod Med 2009;27:62-79.

7. Granot I, Gnainsky Y, Dekel N. Endometrial inflammation and effect on implantation improvement and pregnancy outcome. Reproduction 2012;144:661-8.

8. Akira S, Takeda K, Kaisho T. Toll-like receptors: critical proteins linking innate and acquired immunity. Nat Immunol 2001;2:675-80.

9. Park BS, Lee JO. Recognition of lipopolysaccharide pattern by TLR4 complexes. Exp Mol Med 2013;45:e66.

10. Goldenberg RL, Hauth JC, Andrews WW. Intrauterine infection and preterm delivery. N Engl J Med 2000;342:1500-7.

11. Moustafa S, Joseph DN, Taylor RN, Whirledge S. New models of lipopolysaccharide-induced implantation loss reveal insights into the inflammatory response. Am J Reprod Immunol 2019;81: e13082.

12. Jhamat N, Niazi A, Guo Y, Chanrot M, Ivanova E, Kelsey G, et al. LPS-treatment of bovine endometrial epithelial cells causes differential DNA methylation of genes associated with inflammation and endometrial function. BMC Genomics 2020;21:385.

13. Dimitriadis E, White CA, Jones RL, Salamonsen LA. Cytokines, chemokines and growth factors in endometrium related to implantation. Hum Reprod Update 2005;11:613-30.

14. Massimiani M, Lacconi V, La Civita F, Ticconi C, Rago R, Campagnolo L. Molecular signaling regulating endometrium-blastocyst crosstalk. Int J Mol Sci 2019;21:23.

15. Albelda SM, Buck CA. Integrins and other cell adhesion molecules. FASEB J 1990;4:2868-80.

16. Brown KE, Yamada KM. The role of integrins during vertebrate development. Semin Dev Biol 1995;6:69-77.

17. Humphries JD, Byron A, Humphries MJ. Integrin ligands at a glance. J Cell Sci 2006;119(Pt 19):3901-3.

18. Campbell ID, Humphries MJ. Integrin structure, activation, and interactions. Cold Spring Harb Perspect Biol 2011;3:a004994.

19. Alzamil L, Nikolakopoulou K, Turco MY. Organoid systems to study the human female reproductive tract and pregnancy. Cell Death Differ 2021;28:35-51.

20. You Y, Stelzl P, Zhang Y, Porter J, Liu H, Liao AH, et al. Novel 3D in vitro models to evaluate trophoblast migration and invasion. Am J Reprod Immunol 2019;81:e13076.

21. Uchida H, Maruyama T, Ohta K, Ono M, Arase T, Kagami M, et al. Histone deacetylase inhibitor-induced glycodelin enhances the initial step of implantation. Hum Reprod 2007;22:2615-22.

22. Weimar CH, Post Uiterweer ED, Teklenburg G, Heijnen CJ, Macklon NS. In-vitro model systems for the study of human embryo-endometrium interactions. Reprod Biomed Online 2013;27:461-76.
23. Renaud SJ. Strategies for investigating hemochorial placentation. In: Gupta RC, editor. Reproductive and developmental toxicology. 2nd ed. London: Elsevier; 2017. p. 1259-73.

24. Wang H, Pilla F, Anderson S, Martinez-Escribano S, Herrer I, Moreno-Moya JM, et al. A novel model of human implantation: 3D endometrium-like culture system to study attachment of human trophoblast (Jar) cell spheroids. Mol Hum Reprod 2012;18:33-43.

25. Rao X, Huang X, Zhou Z, Lin X. An improvement of the $2^{\wedge}$ (-delta delta CT) method for quantitative real-time polymerase chain reaction data analysis. Biostat Bioinforma Biomath 2013;3:71-85.

26. Mor G, Cardenas I, Abrahams V, Guller S. Inflammation and pregnancy: the role of the immune system at the implantation site. Ann NY Acad Sci 2011;1221:80-7.

27. Kitazawa J, Kimura F, Nakamura A, Morimune A, Takahashi A, Takashima A, et al. Endometrial immunity for embryo implantation and pregnancy establishment. Tohoku J Exp Med 2020;250: 49-60.

28. Dekel N, Gnainsky Y, Granot I, Racicot K, Mor G. The role of inflammation for a successful implantation. Am J Reprod Immunol 2014; 72:141-7.

29. Chishima F, Nakajima T, Nakao T, Hayashi C, Ichikawa G, Komatsu A, et al. The inflammatory process and successful implantation. J Mamm Ova Res 2017;34:75-81.

30. Park DW, Yang KM. Hormonal regulation of uterine chemokines and immune cells. Clin Exp Reprod Med 2011;38:179-85.

31. Bashiri A, Halper KI, Orvieto R. Recurrent implantation failure-update overview on etiology, diagnosis, treatment and future directions. Reprod Biol Endocrinol 2018;16:121.

32. Akopians AL, Pisarska MD, Wang ET. The role of inflammatory pathways in implantation failure: chronic endometritis and hydrosalpinges. Semin Reprod Med 2015;33:298-304.

33. Deb K, Chaturvedi MM, Jaiswal YK. Gram-negative bacterial LPS induced poor uterine receptivity and implantation failure in mouse. alterations in IL-1beta expression in the preimplantation embryo and uterine horns. Infect Dis Obstet Gynecol 2005;13: 125-33.

34. Stowell NC, Seideman J, Raymond HA, Smalley KA, Lamb RJ, Egenolf DD, et al. Long-term activation of TLR3 by poly $(\mathrm{l}: \mathrm{C})$ induces inflammation and impairs lung function in mice. Respir Res 2009;10:43.

35. Montazeri M, Sanchez-Lopez JA, Caballero I, Maslehat Lay N, Elliott S, Lopez-Martin S, et al. Activation of Toll-like receptor 3 reduces actin polymerization and adhesion molecule expression in endometrial cells, a potential mechanism for viral-induced implantation failure. Hum Reprod 2015;30:893-905.

36. Aboussahoud W, Bruce C, Elliott S, Fazeli A. Activation of Toll-like receptor 5 decreases the attachment of human trophoblast cells 
to endometrial cells in vitro. Hum Reprod 2010;25:2217-28.

37. Mokhtari S, Mahdavi AH, Hajian M, Kowsar R, Varnosfaderani SR, Nasr-Esfahani MH. The attenuation of the toxic effects of LPS on mouse pre-implantation development by alpha-lipoic acid. Theriogenology 2020;143:139-47.

38. Palsson-McDermott EM, O'Neill LA. Signal transduction by the lipopolysaccharide receptor, Toll-like receptor-4. Immunology 2004;113:153-62.

39. Gierman LM, Stodle GS, Tangeras LH, Austdal M, Olsen GD, Follestad T, et al. Toll-like receptor profiling of seven trophoblast cell lines warrants caution for translation to primary trophoblasts. Placenta 2015;36:1246-53.

40. Aboussahoud W, Aflatoonian R, Bruce C, Elliott S, Ward J, Newton $\mathrm{S}$, et al. Expression and function of Toll-like receptors in human endometrial epithelial cell lines. J Reprod Immunol 2010;84:4151.

41. Hayman EG, Pierschbacher MD, Suzuki S, Ruoslahti E. Vitronectin: a major cell attachment-promoting protein in fetal bovine serum. Exp Cell Res 1985;160:245-58.

42. Bas S, James RW, Gabay C. Serum lipoproteins attenuate macrophage activation and Toll-like receptor stimulation by bacterial lipoproteins. BMC Immunol 2010;11:46.

43. Helige C, Ahammer H, Hammer A, Huppertz B, Frank HG, Dohr G. Trophoblastic invasion in vitro and in vivo. similarities and differences. Hum Reprod 2008;23:2282-91.

44. Merviel P, Challier JC, Carbillon L, Foidart JM, Uzan S. The role of integrins in human embryo implantation. Fetal Diagn Ther 2001; 16:364-71.

45. Guo Y, van Schaik T, Jhamat N, Niazi A, Chanrot M, Charpigny G, et al. Differential gene expression in bovine endometrial epithelial cells after challenge with LPS: specific implications for genes involved in embryo maternal interactions. PLoS One 2019;14: e0222081.

46. Luk J, Seval Y, Kayisli UA, Ulukus M, Ulukus CE, Arici A. Regulation of interleukin-8 expression in human endometrial endothelial cells. a potential mechanism for the pathogenesis of endometriosis. J Clin Endocrinol Metab 2005;90:1805-11.

47. Sheldon IM, Roberts MH. Toll-like receptor 4 mediates the response of epithelial and stromal cells to lipopolysaccharide in the endometrium. PLoS One 2010;5:e12906.

48. Wang J, Yan X, Nesengani LT, Ding H, Yang L, Lu W. LPS-induces IL-6 and IL-8 gene expression in bovine endometrial cells "through DNA methylation". Gene 2018;677:266-72.

49. Miller JE, Monsanto SP, Ahn SH, Khalaj K, Fazleabas AT, Young SL, et al. Interleukin-33 modulates inflammation in endometriosis. Sci Rep 2017;7:17903.

50. PrabhuDas M, Bonney E, Caron K, Dey S, Erlebacher A, Fazleabas A, et al. Immune mechanisms at the maternal-fetal interface: perspectives and challenges. Nat Immunol 2015;16:328-34.

51. Moreno-Moya JM, Ramirez L, Vilella F, Martinez S, Quinonero A, Noguera I, et al. Complete method to obtain, culture, and transfer mouse blastocysts nonsurgically to study implantation and development. Fertil Steril 2014;101:e13.

52. Bin Ali R, van der Ahe F, Braumuller TM, Pritchard C, Krimpenfort $P$, Berns A, et al. Improved pregnancy and birth rates with routine application of nonsurgical embryo transfer. Transgenic Res 2014; 23:691-5.

53. Kim J, Lee J, Jun JH. Advantages of the outgrowth model for evaluating the implantation competence of blastocysts. Clin Exp Reprod Med 2020;47:85-93. 